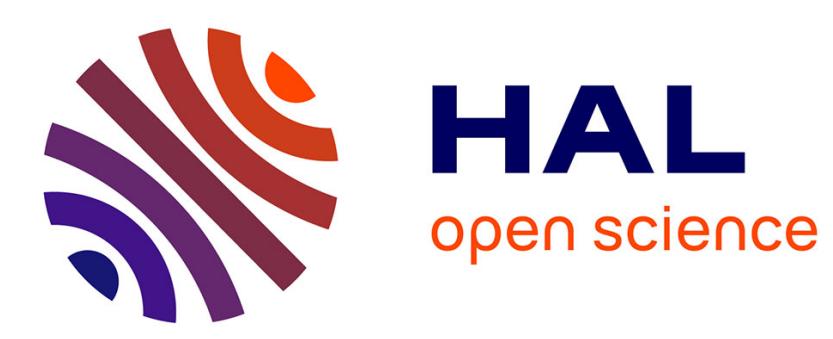

\title{
Bow-wave-like hydraulic jump and horseshoe vortex around an obstacle in a supercritical open channel flow
}

\author{
Emmanuel Mignot, Nicolas Riviere
}

\section{To cite this version:}

Emmanuel Mignot, Nicolas Riviere. Bow-wave-like hydraulic jump and horseshoe vortex around an obstacle in a supercritical open channel flow. Physics of Fluids, 2010, 22, pp.117105. 10.1063/1.3505013 . hal-00746687

\section{HAL Id: hal-00746687 \\ https://hal.science/hal-00746687}

Submitted on 24 Jan 2013

HAL is a multi-disciplinary open access archive for the deposit and dissemination of scientific research documents, whether they are published or not. The documents may come from teaching and research institutions in France or abroad, or from public or private research centers.
L'archive ouverte pluridisciplinaire HAL, est destinée au dépôt et à la diffusion de documents scientifiques de niveau recherche, publiés ou non, émanant des établissements d'enseignement et de recherche français ou étrangers, des laboratoires publics ou privés. 


\title{
Bow-wave-like hydraulic jump and horseshoe vortex around an obstacle in a supercritical open channel flow
}

\author{
E. Mignot and N. Riviere \\ LMFA, CNRS-Universite de Lyon, INSA de Lyon, Bat. Joseph Jacquard, 20 avenue A. Einstein, \\ 69621 Villeurbanne Cedex, France
}

(Received 16 December 2009; accepted 22 September 2010; published online 11 November 2010)

\begin{abstract}
The objective of the present paper is to characterize the interaction of a supercritical open channel flow with an emerged, rectangular shaped obstacle. Upstream from the obstacle, two main flow structures are observed: (i) a hydraulic jump in the near-surface region and (ii) a horseshoe vortex in the near-bed region. Both flow structures are detached upstream from the obstacle and two main flow types occur regarding their relative detachment lengths. For high Reynolds numbers (turbulent regime), the detachment length of the hydraulic jump exceeds the one of the horseshoe vortex; this flow type is named "breaking type." Oppositely, for lower Reynolds numbers (laminar regime), the detachment length of the horseshoe vortex exceeds the one of the hydraulic jump, and the flow type is named "separation type." Experimental measurements based on particle deposition and colored streamline deflection are used to detect the location and shape of the detachment curves. We showed that for both flow types, the shape of the hydraulic jump curve is hyperbolic, constrained by two asymptotes following the Froude angle on each side of the obstacle. On the other hand, the shape of the horseshoe vortex curve depends on the flow type; it is hyperbolic in the separation type and elliptic in the breaking type. The dimensional analysis reveals that three parameters can influence the detachment curves: the upstream Froude and Reynolds numbers and the upstream water depth normalized by the obstacle thickness. The influence of these parameters is investigated experimentally on a water table for both flow types. (c) 2010 American Institute of Physics.

[doi:10.1063/1.3505013]
\end{abstract}

\section{INTRODUCTION}

In the literature, many studies describing the flow patterns that occur as subsonic air flows and interact with emerging obstacles in wind tunnels are available. As the incoming flow approaches the obstacle, the adverse pressure gradient between the upstream flow and the flow at the obstacle leads to a boundary layer separation in the near-bottom region. Consequently, a horseshoe vortex structure is observed at the toe of the obstacle on its upstream side. If the velocity of the incoming flow is low enough, a multiple vortices structure takes place, while if the velocity (and the Reynolds number) of the flow increases, a single vortex is observed; if the velocity keeps on increasing, no fully developed vortex is observed. ${ }^{1}$ Recently, Pattenden et al. ${ }^{2}$ proposed a fine description of the horseshoe vortex using a combination of oil flow visualization, particle image velocimetry, and surface pressure measurement. In order to characterize the distance between the detachment of the boundary layer and the obstacle, noted $\lambda$ herein, Baker $^{3}$ derived the following normalized relation obtained from dimensional analysis (with $R$ as the obstacle typical dimension and $\operatorname{Re}_{\delta^{*}}$ as the Reynolds number based on the boundary layer displacement length $\delta^{*}$ ):

$$
\frac{\lambda}{R}=f\left(\operatorname{Re}_{\delta^{*}}, \frac{\delta^{*}}{R}\right) .
$$

Ballio et $a{ }^{4}{ }^{4}$ collected all available experimental data in order to evaluate the influence of these parameters. They observed that for $10^{3}<\operatorname{Re}_{\delta^{*}}<10^{7}$, the Reynolds number $\operatorname{Re}_{\delta^{*}}$ has no effect on the normalized horseshoe vortex detachment length $\lambda / R$.

Concerning open channel water flows, most studies are dedicated to investigate the flow patterns upstream from obstacles in subcritical flow regimes for bridge pier scour application purposes. As for air flows, a horseshoe vortex is observed at the toe of the obstacle as a consequence of the wall boundary layer separation. Authors such as Dargahi ${ }^{5}$ and Sahin et al. ${ }^{6}$ showed that depending on the Reynolds number of the inflow, a single vortex or a multiple vortices structure is observed within the horseshoe vortex. Graf and Yulistiyanto, ${ }^{7}$ Ahmed and Rajaratnam, ${ }^{8}$ and Roulund et al. ${ }^{9}$ described the flow patterns and shear stress fields upstream from the obstacle and within the horseshoe vortex. They showed that the streamwise velocity decreases as the water depth increases before reaching the obstacle, and the vertical profile of streamwise velocity tends toward uniformity. Moreover, reverse streamwise velocities are measured in the near-bed region within the horseshoe vortex. Furthermore, the measured vertical velocity is downward in the region affected by the obstacle and its magnitude increases when approaching the obstacle. Roulund et al. ${ }^{9}$ and Sadeque et $a l{ }^{10}$ experimentally showed that the bed shear stress is negative away from the obstacle, goes to zero, and becomes positive when approaching the obstacle. The location where the bed shear stress reaches a zero magnitude corresponds to the separation point of the boundary layer and thus the upstream limit of the horseshoe vortex. ${ }^{9}$ However, only few 
data related to the location of the toe of the horseshoe vortex are available. Dargahi ${ }^{5}$ and Roulund et al. ${ }^{9}$ each proposed a single measurement of such location for one flow configuration.

Works on detached shock waves upstream bluff-bodies have been undertaken for decades, essentially for aerodynamic purposes. For instance, Shapiro ${ }^{11}$ provided a comprehensive description of shocks around slender or bluff bodies embedded in a supersonic gas flow. For bluff bodies, a detached, bow shaped, shock wave forms upstream the body. Moeckel $^{12}$ developed an analytical model predicting the shape and location of this detached shock. As confirmed by experimental results, this author showed that the normalized detachment length of the shock wave decreases as Mach number increases. Experimental (e.g. Ref. 13) or numerical (e.g., Ref. 14) investigations are still performed on this topic.

Finally, a few studies characterize the interactions between a supercritical water inflow and an obstacle. Forbes and Schwartz ${ }^{15}$ applied a theoretical power-series approach to characterize the hydraulic jump in front of the obstacle for two limiting cases: infinite and critical Froude number, but without considering neither the presence of the horseshoe vortex nor the jump width. Jiang and Smith ${ }^{16}$ investigated the case of a free surface shallow water flow over an isolated bump using numerical modeling. For a supercritical inflow with relatively limited Froude number, they found that facing a sufficiently elevated obstacle, a stationary shock takes place in front of the obstacle. The shape of the computed shock is a bow wave near the centerline and a V-wave further away. Near the centerline, the shock is perpendicular to the flow axis, and past the jump, the flow becomes subcritical and is deflected away from the centerline. Further from the centerline, the supercritical flow experiences an oblique jump at the trailing edge of the V-wave and the flow downstream from the jump remains supercritical as predicted by Ippen. ${ }^{17}$ Defina and Susin ${ }^{18}$ roughly described the flow pattern upstream from the obstacle and also reported the occurrence of a detached hydraulic jump around the obstacle. However, in these studies, the authors did not report the presence of a horseshoe vortex and did not discuss the characteristics of the jump (width of the jump and distance of detachment).

An experimental study is performed at LMFA (Lyon, France) in order to quantitatively investigate the interaction between a supercritical water inflow and an emerging obstacle. In one section, we describe the observed flow types. Then, the dimensional analysis is used to identify the parameters, which may influence the flow structure detachment lengths. In another section, the experimental setup used for measuring the location of the main flow structures is presented for each flow type. Finally, the influence of the normalized parameters on the occurrence of the flow types and on the location of the main flow structures is analyzed in details.

\section{FLOW DESCRIPTION}

As a supercritical open channel flow interacts with an obstacle deposited on the bottom and emerging above the free surface, a complex flow structure develops. On the one hand, the incoming flow decelerates, reaches a subcritical regime through a hydraulic jump, and keeps on decelerating until reaching the obstacle with no streamwise velocity. This velocity reduction creates a discontinuous water depth increase in the hydraulic jump followed by a continuous depth increase up to the obstacle. Moreover, the adverse pressure gradient in the streamwise direction leads to a boundary layer separation in the near-bed region upstream from the obstacle. This separation gives rise to a horseshoe vortex, as discussed in the literature review. On the other hand, a transverse water depth gradient appears, related to the larger water depth in front of the obstacle than on its sides. This gradient causes the flow to move around the obstacle.

To summarize, two main flow structures were observed on our experimental setup upstream from the obstacle: a hydraulic jump in the near-surface region and a horseshoe vortex in the near-bed region. As expected, both flow structures are detached upstream from the obstacle, and it is observed that their relative detachment lengths vary regarding the inflow and obstacle parameters. We decided to classify the flow configurations into the three following flow types.

- For a laminar inflow, the detachment length for the toe of the horseshoe vortex $\left(\lambda_{H}\right)$ is larger than for the toe of the hydraulic jump $\left(\lambda_{J}\right)$. The uniform inflow first reaches the vortex, which acts as a positive step [Fig. 1(a)]. The flow remains in supercritical regime with a water depth slightly increased $\left[h_{1}^{\prime}>h_{1}\right.$ in Fig. 1(a)] and a velocity slightly decreased. The flow then reaches the hydraulic jump and passes from supercritical to subcritical regime $\left(h_{2}^{\prime}\right)$. Finally, the water depth increases and reaches its maximum value $\left(Z_{o}\right)$ on the upstream face of the obstacle. This flow type is named "separation flow type." The streak-line photograph in Fig. 1(a) shows that the oncoming flow is laminar.

- For a turbulent inflow, the detachment length for the toe of the horseshoe vortex is lower than for the toe of the hydraulic jump. In this case, the supercritical uniform flow first reaches the hydraulic jump where it passes to subcritical regime $\left[h_{2}\right.$ in Fig. 1(b)]. The subcritical flow then reaches the horseshoe vortex and moves over it (as over a positive step). Finally, the water depth keeps on increasing until reaching the obstacle. This type is named "breaking flow type." The streak-line photograph in Fig. 1(b) tends to show that the oncoming flow is turbulent.

- For an inflow regime at the transition between laminar and turbulent, the upstream flow is laminar with turbulent bursts, which increase in size as they are transported along the flow. As these bursts reach the obstacle region, the location of the boundary layer separation oscillates in time, and this separation is sometimes located upstream from the hydraulic jump (as in the separation type) and sometimes downstream from the jump (as in the breaking type). When the boundary layer separation passes from one location to the other, the water depth at the toe of the hydraulic jump is strongly affected (it varies between $h_{1}$ and $h_{1}^{\prime}$ 

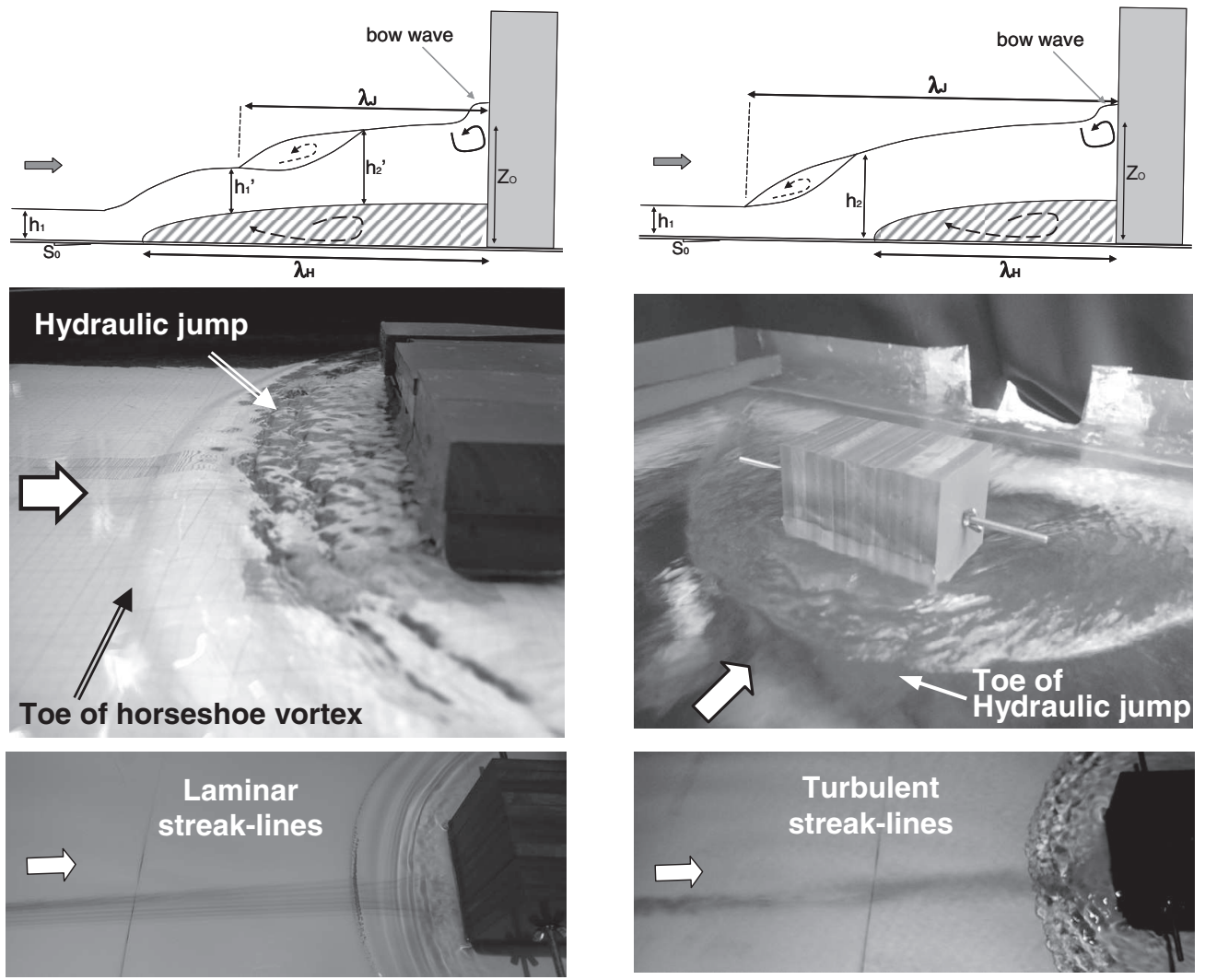

a) Separation type

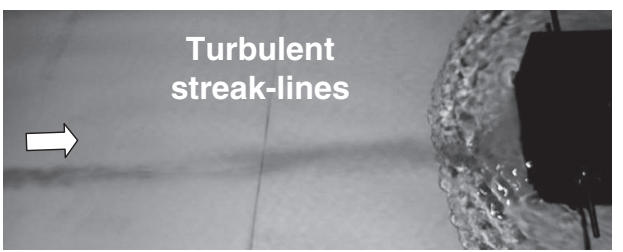

b) Breaking type

FIG. 1. From top to bottom: scheme (side views), photograph, and near-bed streamline photograph of a separation type flow (a) and a breaking type flow (b).

in Fig. 1 sketches), and the location of the jump then also varies. Consequently, the flow pattern oscillates between the breaking and separation types with a finite-amplitude oscillation; this flow type is thus named "unstable."

It is well known that in laminar flow, the boundary layer separation is enhanced compared to a turbulent flow. Indeed, in turbulent regime, the Reynolds stresses can transfer momentum from rapid flow regions away from the wall toward near-wall regions with lower velocity; this transfer prevents excessive flow deceleration in moderate pressure gradients and thus moves the boundary layer separation further downstream. ${ }^{19}$ When collecting the available boundary separation length data upstream from obstacles in wind tunnels with subcritical inflows, Ballio et al., ${ }^{4}$ in their Fig. 3, showed that the horseshoe vortex detachment length is greater for the laminar inflow regime than for the turbulent inflow regime. The higher horseshoe vortex detachment lengths in separation type than in breaking type presented herein is in fair agreement with theses observations.

The previous flow description was simplified in order to sort the flow configurations into a typology. Secondary phenomena were actually observed for some conditions. For instance, a surface bow wave is present around the stagnation point, ${ }^{20}$ which can interact with the jump. ${ }^{21}$

\section{DIMENSIONAL ANALYSIS}

Baker $^{3}$ first proposed a dimensional analysis for the detachment length of the horseshoe vortex upstream from an obstacle in wind tunnel conditions [see Eq. (1)]. Following this approach, in the present paper, we adapt this dimensional analysis to the horseshoe vortex and the hydraulic jump detachment lengths $\lambda$ in open channel conditions. The flow and obstacle parameters that can affect $\lambda$ are the water density $\rho$ and dynamic viscosity $\mu$, the gravity acceleration $g$, the surface tension $\sigma$ at the water/air interface, the equivalent bed roughness $k_{s}$, the inflow bulk velocity $U$ and uniform water depth $h_{1}$, and finally the width of the obstacle $R$,

$$
\lambda=f\left(\rho, \mu, g, \sigma, k_{s}, U, h_{1}, R\right) .
$$

Equation (2) may be normalized as

$$
\frac{\lambda}{R}=f\left(\text { Fr, } \frac{h_{1}}{R}, \operatorname{Re}, \frac{k_{s}}{R}, \mathrm{We}\right),
$$

where $\mathrm{Fr}=U /\left(g h_{1}\right)^{1 / 2}, \mathrm{Re}=4 \rho U h_{1} / \mu$, and $\mathrm{We}=\rho U^{2} h_{1} / \sigma$ are the inflow Froude, Reynolds, and Weber numbers, respectively. In Eq. (3), either the Weber number We or Reynolds number Re can be replaced by the Morton number Mo, which is a composition of We, Re, and Fr, ${ }^{22,23}$

$$
\mathrm{Mo}=\frac{\mathrm{We}^{3}}{\operatorname{Fr}^{2}(\operatorname{Re} / 4)^{4}}=\frac{g \mu^{4}}{\rho \sigma^{3}} .
$$


Mo depends only on the physical properties of the fluids. Hence, Mo does not vary with the flow characteristics such as discharge or water depth contrary to Re and We. For given fluids-such as water and air as in the present case-Mo remains constant for all flow configurations. Consequently, replacing $\mathrm{Re}$ or We by Mo reduces the number of dimensionless independent parameters which must be accounted for in our parametric study.

Observations of the influence of both Re and We on the flow pattern, detailed in the following sections of this paper, reveal that Re has a main influence on the flow pattern and should be included in the dimensional analysis. We thus decided to replace We by Mo, and Eq. (3) becomes

$$
\frac{\lambda}{R}=f\left(\text { Fr, } \frac{h_{1}}{R}, \operatorname{Re}, \frac{k_{s}}{R}, \text { Mo }\right),
$$

where Mo is fixed for a couple of fluids. Moreover, the friction velocity $u^{*}$ may be computed using the uniform flow condition

$$
u^{*}=\sqrt{g h_{1} S_{0}}
$$

where $S_{0}$ is the bed slope. The order of magnitude of the friction velocity is about $u^{*} \approx 2-3 \mathrm{~cm} / \mathrm{s}$ for all tested configurations. As the bottom of the water table is made of glass, with a typical equivalent roughness $k_{s}=10^{-7} \mathrm{~m}$, the roughness Reynolds number $k_{s}^{+}=k_{s} u^{*} / \nu \approx 2-3 \times 10^{-3}$. Given that $k_{s}^{+} \ll 1$, our flows are in fully smooth regime, the roughness elements are confined within the viscous sublayer, and the roughness parameter has no influence on the flow.

Furthermore, if we consider the free surface as an interface between pure water and air, the Morton number value, which depends only on the fluid characteristics, is fixed with $\mathrm{Mo}=2.4 \times 10^{-11}$ and will remain equal for any change of scale. In such conditions, Eq. (5) reads

$$
\frac{\lambda}{R}=f\left(\operatorname{Fr}, \frac{h_{1}}{R}, \operatorname{Re}\right) \text {. }
$$

The detachment lengths of the hydraulic jump and the horseshoe vortex normalized by the obstacle width thus depend on three parameters. The next sections are dedicated to the analysis of the influence of these parameters on the detachment of the flow structures.

\section{EXPERIMENTAL SETUP}

The experiments are performed in a smooth, transparent, $L=118 \mathrm{~cm}$ long, and $b=74.5 \mathrm{~cm}$ wide water table with adjusting slope. A pump provides water to the flume from a storage tank. The water reaches the water table through a stilling basin and perforated plates. The channel section and slope remain constant over the water table. The length of the water table ensures to reach the flow uniformity before getting to the test section which starts $50 \mathrm{~cm}$ downstream from the stilling basin (that is more than 100 times the upstream water depth) according to Ranga Raju et al. ${ }^{24}$ observations. The combination of slope and discharge then defines the uniform flow depth $h_{1}$. Discharge $Q$ is measured by an electromagnetic flowmeter (Promag 50, Endress Hauser, Huningue, France, uncertainty $0.01 \mathrm{~L} / \mathrm{s}$ ). The uniform flow depth $h_{1}$ is

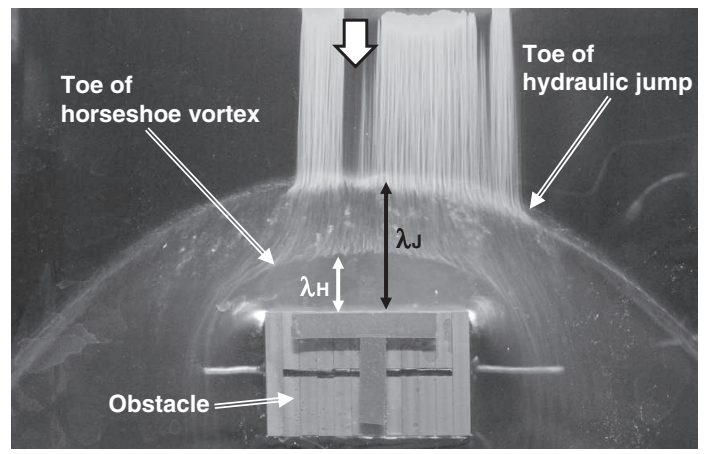

FIG. 2. Typical photograph of a breaking type flow.

measured manually using a digital limnimeter (uncertainty of $0.2 \mathrm{~mm}$ ). The emerging obstacle is placed on the water table at a distance of about $90 \mathrm{~cm}$ downstream from the stilling basin. A referencing tape fixed on the bottom of the water table (see Figs. 2 and 3) ensures the obstacle alignment with regard to the inflow axis. The obstacle is made of several 2 or $10 \mathrm{~mm}$ thick individual squared plastic plates maintained together [see Fig. 1(b)]. Changing the number of plates included in the obstacle enables a rapid and precise modification of the obstacle width $(R)$. When leaving the water table, the flow is collected in the downstream tank where a pump allows a recirculation of the water. The experimental setup is then placed within an opaque tent and a light source is introduced below the upstream tank in order to control the lightning. A $14 \mathrm{~mm}$ opening camera is fixed to the water table support just below the obstacle. The camera is controlled by a computer located outside the tent. The measurement methodology is then adapted for each flow type: separation and breaking.

\section{A. Measurement methodology for breaking type flows}

Figure 2 shows a typical photograph of a breaking type flow obtained in the conditions described below. The method aiming at detecting the toe of the horseshoe vortex is adapted from the classical oil flow visualization method used to detect the lines of separation and attachment in air flow conditions (see, for instance, Refs. 3, 1, and 2). When the flow and obstacle parameters $(Q, R$, and water table slope) are fixed to their required values, the pump is stopped, the water table is dried, and a thick transverse layer of water-base painting is deposited on the water table upstream from the obstacle. The pump is then switched on again. When steady conditions are reached, the supercritical inflow carries painting along nearbed streamlines through the hydraulic jump, and these colored streamlines are deflected laterally along the toe of the horseshoe vortex; they finally move around the obstacle (see Fig. 2). Moreover, the instability of the free surface in the hydraulic jump causes a strong water depth gradient fluctuation, which creates a strong light reflection toward the camera. Hence, the roller of the hydraulic jump is revealed on the photograph by a brighter region (see Fig. 2). A comparison with visual detection of the toe of the hydraulic jump confirmed that the upstream boundary of the bright region corresponds to the toe of the hydraulic jump. Photographs of the 


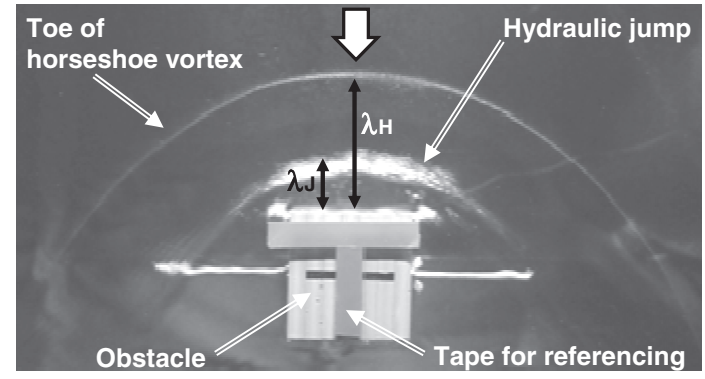

FIG. 3. Typical photograph of a separation type flow.

flow are finally taken in order to detect both the location of the hydraulic jump and of the horseshoe vortex.

\section{B. Measurement methodology for separation type flows}

Figure 3 shows a typical photograph of a separation type flow obtained in the conditions described below. On the one hand, the methodology used to detect the hydraulic jump is similar to the breaking flow type, the brighter region corresponding to the jump appears clearly in Fig. 3. On the other hand, solid particles (Iriodin 500 "Bronze," $d=10-60 \mu \mathrm{m}$ ) are added to the water. These particles have a density of 3 . Due to their small size, these particles act as tracers with limited relative velocity in the supercritical inflow, but due to their high density, they settle when the velocity reduces significantly. At the boundary layer separation, the streamwise velocity becomes locally damped, especially in the near-bed region as the upstream flow intersects the return flow. The shear stress is suddenly strongly reduced (as measured by Roulund et $a l .{ }^{9}$ ), and we reach the so-called "zero wall friction" location. The solid particles deposit forming a light curve on the photograph referred to as "toe of horseshoe vortex" in Fig. 3. A comparison of this detection method with the painting colored streamline visualization method described above confirmed the validity of this technique to detect the toe of the horseshoe vortex. The interest of such particle settling method compared to painting flow visualization is that the flow does not have to be stopped during the experiment. However, this particle settling method cannot be used in breaking type flows as the horseshoe vortex takes place downstream from the hydraulic jump and large scale turbulent structures created by the hydraulic jump tend to disperse the solid particles while settling.

\section{Image analysis}

For each flow configuration, photographs are taken in controlled conditions. Image analysis programs are written in order to locate semiautomatically the curves corresponding to the toe of the horseshoe vortex and of the hydraulic jump. As a first step, the user locates both corners of the obstacle upstream face, and the reference frame is defined at the center of this face. The programs then differ between both flow types.

For the separation type, a maximum brightness gradient method is applied in order to locate the curve corresponding
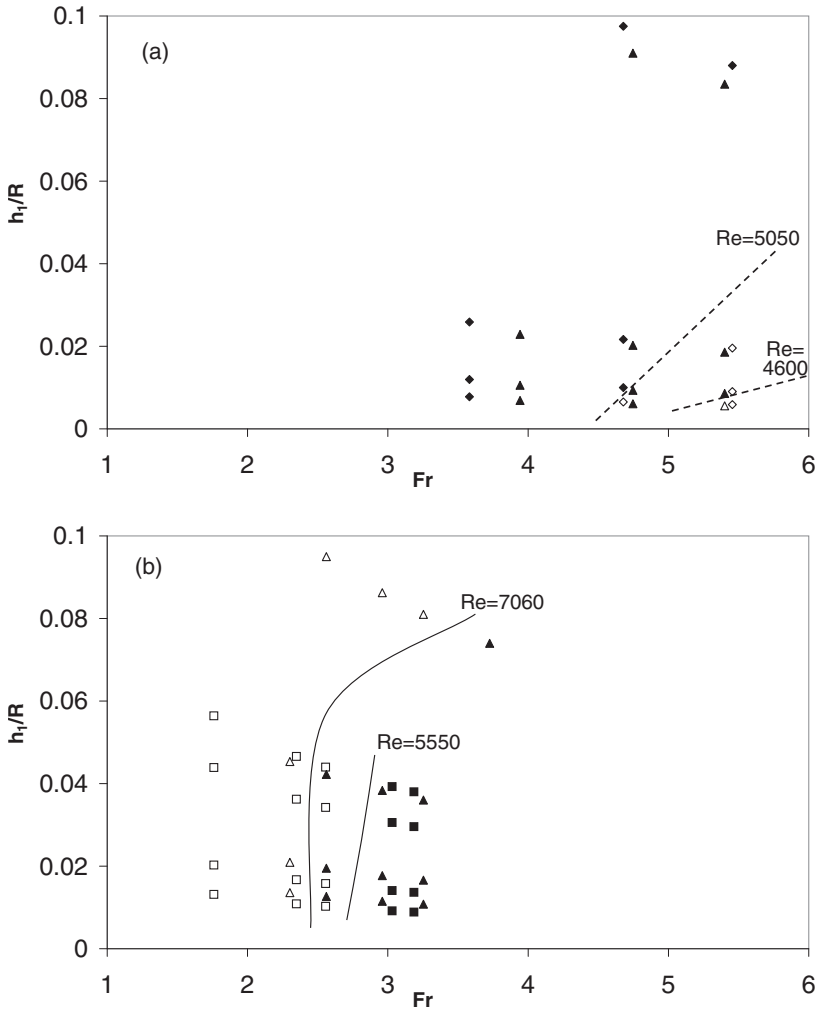

FIG. 4. (a) Conditions of occurrence of separation (black symbol) and unstable (white symbol) flow types with $(--)=$ limit between both types for $\mathrm{Re}=4600$ (triangles) and $\mathrm{Re}=5050$ (lozenges). (b) Conditions of occurrence of breaking (black symbol) and unstable (white symbol) flow types with $(-)=$ limit between both types for $\operatorname{Re}=5550$ (squares) and $\operatorname{Re}=7060$ (triangles). Data for the range of investigated parameter values $\left(1.3<F_{r}<6,3000<\operatorname{Re}<10000\right.$, and $\left.0.005<h_{1} / R<0.1\right)$.

to the toe of the horseshoe vortex where particles have started to deposit (see Fig. 3). Moreover, in front of the obstacle, a brightness threshold is applied in order to detect the perimeter of the hydraulic jump roller region. When filtered, the upstream part of this perimeter corresponds to the toe of the hydraulic jump. The uncertainty of the curve detection estimated from photographs is the following: $2.5 \mathrm{~mm}$ for the horseshoe vortex and $3.2 \mathrm{~mm}$ for the hydraulic jump.

For the breaking type, a computer-aided manual detection method is applied: the user is invited to click on both curves along their development lengths. The uncertainty for the curve detections is the following: $2.5 \mathrm{~mm}$ for the horseshoe vortex and $4 \mathrm{~mm}$ for the hydraulic jump.

\section{OCCURRENCE OF BOTH FLOW TYPES}

As a first step, a large number of flow configurations were observed visually by varying one parameter from Eq. (7) at a time and detecting the flow type that developed. The condition of occurrence of the three flow types is presented on Fig. 4 and detailed below. Figure 4 reveals that the Reynolds number is the key parameter that most influences the flow type. For high Re configurations, the streamlines located in the near-bed region are turbulent, and the breaking type flow is more likely to take place while for low Re con- 
TABLE I. Experimental data for the three separation $(S)$ and the three breaking $(B)$ series. Values in bracket are the intervals of nonfixed parameters values.

\begin{tabular}{lcccccc}
\hline \hline Case & $\begin{array}{c}Q \\
(\mathrm{~L} / \mathrm{s})\end{array}$ & $\begin{array}{c}h_{1} \\
(\mathrm{~mm})\end{array}$ & $\mathrm{Fr}$ & $\mathrm{Re}$ & $h_{1} / R$ & $\beta=\sqrt{\mathrm{Fr}^{2}-1}$ \\
\hline$S 1$ & 0.78 & 2.5 & 2.67 & 4188 & {$[0.014 ; 0.042]$} & 2.48 \\
$S 2$ & 0.61 & {$[1.97-3.42]$} & {$[1.3-2.99]$} & 3275 & 0.035 & {$[0.83-2.82]$} \\
$S 3$ & {$[0.306-0.862]$} & {$[1.48-2.91]$} & 2.33 & {$[2153-4628]$} & 0.046 & 2.10 \\
$B 1$ & 1.5 & 3.9 & 2.64 & 8054 & {$[0.023-0.056]$} & 2.44 \\
$B 2$ & 1.5 & {$[2.86-4.2]$} & {$[2.36-4.2]$} & 8054 & 0.035 & {$[2.14-4.08]$} \\
$B 3$ & {$[1.5-2.2]$} & {$[4.24-5.47]$} & 2.33 & {$[8054-11812]$} & 0.046 & 2.10 \\
\hline \hline
\end{tabular}

figurations, these streamlines are in laminar regime and mostly separation type takes place. We observed the following.

- First, for $\operatorname{Re}<4600$, all flow configurations are in separation type.

- Oppositely, for Re $>8000$, all flow configurations are in breaking type.

- Finally, for intermediate Reynolds numbers, three relevant situations are observed: (i) for $4600<\mathrm{Re}$ $<5200$, the flow may be in separation type or Unstable type regarding the value of Fr and the ratio $h_{1} / R$ [Fig. 4(a)]; (ii) for $5400<\operatorname{Re}<8000$, the flow may be in breaking type or unstable type regarding the value of Fr and $h_{1} / R$ [Fig. 4(b)]; and (iii) for $\mathrm{Re} \approx 5300$, only the unstable type was observed.

\section{MEASUREMENT OF DETACHMENT CURVES}

For each flow type ( $S$ for separation and $B$ for breaking), three data sets are investigated experimentally (see Table I) by keeping two of the normalized parameters from Eq. (7) constant and varying the third one. For $S 1$ and $B 1$, Fr and Re are kept constant, while $h_{1} / R$ varies; for $S 2$ and $B 2$, Re and $h_{1} / R$ are kept constant, while Fr varies; and for $S 3$ and $B 3$, Fr and $h_{1} / R$ are kept constant, while Re varies. This permits to investigate the influence of each normalized parameter individually on the detachment curves.

Figure 5(a) shows that in the separation type, the detachment curve of the toe of the horseshoe vortex and the hydraulic jump can be fitted using an adapted version of the hyperbolic curve proposed by Moeckel. ${ }^{12}$ Indeed, the hydraulic jump is perpendicular to the flow axis in front of the obstacle and reaches the Froude angle (equivalent to the well known Mach angle) far on each side. The adverse pressure gradient caused by the water depth increase due to this hydraulic jump will force the boundary layer to detach slightly upstream from this hydraulic jump along the whole jump curve [see Fig. 5(a)]. Consequently, the general shape of the horseshoe vortex curve will be similar to the hydraulic jump curve. On the other hand, Fig. 5(b) confirms that for the breaking type, the detachment curve of the toe of the hydraulic jump can still be fitted using an adapted version of the curve proposed by Moeckel. ${ }^{12}$ However, at the toe of the horseshoe vortex, the flow is in subcritical regime, and the boundary layer detachment is produced by the water depth increase from the downstream side of the hydraulic jump to the obstacle [see Fig. 1(b)]. This water depth increase only exists in front of the obstacle and rapidly vanishes on its sides. Consequently, as previously observed (see, for instance, Refs. 25 and 10), the horseshoe vortex curve will be perpendicular to the flow axis in front of the obstacle and will rapidly tend to be parallel to this axis on the sides of the obstacle [see Fig. 5(b)]. The detachment curve of the toe of the horseshoe vortex is "U-shaped" (see Ref. 20) and must then be fitted by an ellipse.
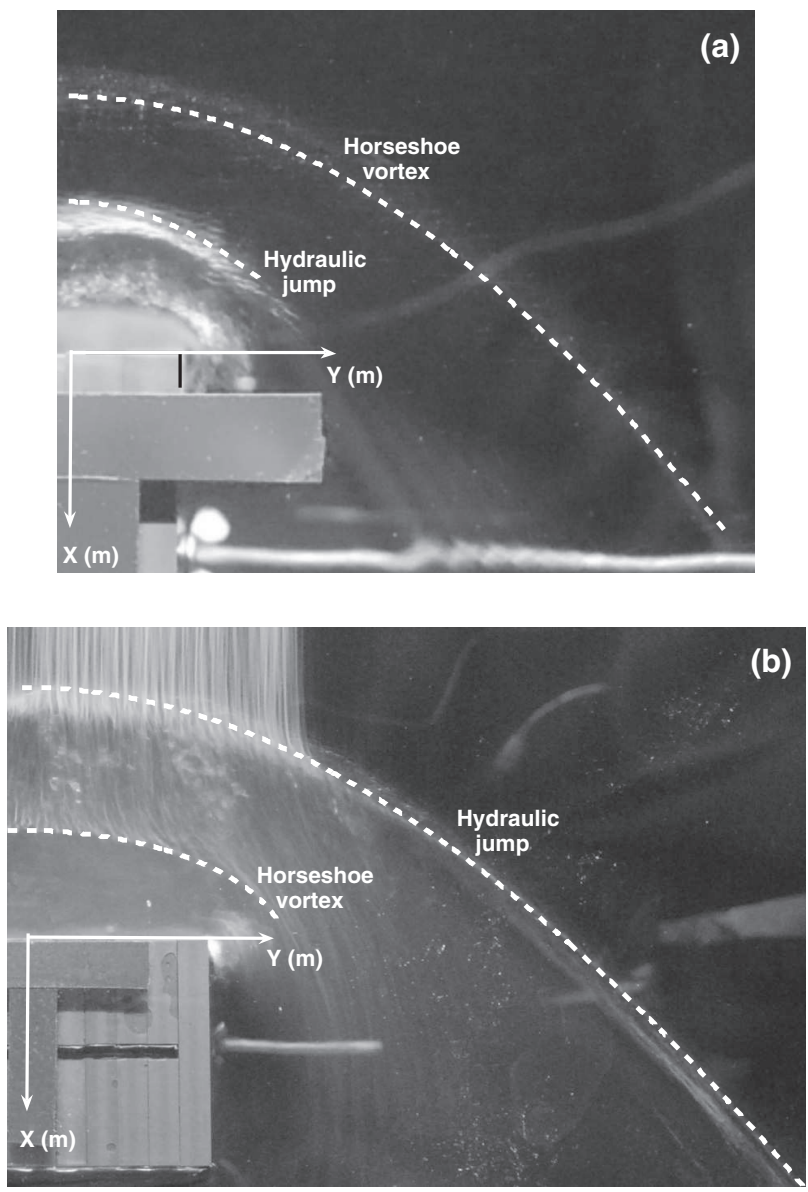

FIG. 5. Photograph analysis for a separation type (a) and breaking type (b) flow. In (a), the white dotted lines are the fitted curve using Eq. (10). In (b), the hydraulic jump white dotted line is the fitted curve using Eq. (10), while the horseshoe vortex white dotted line is the fitted curve using Eq. (12). 


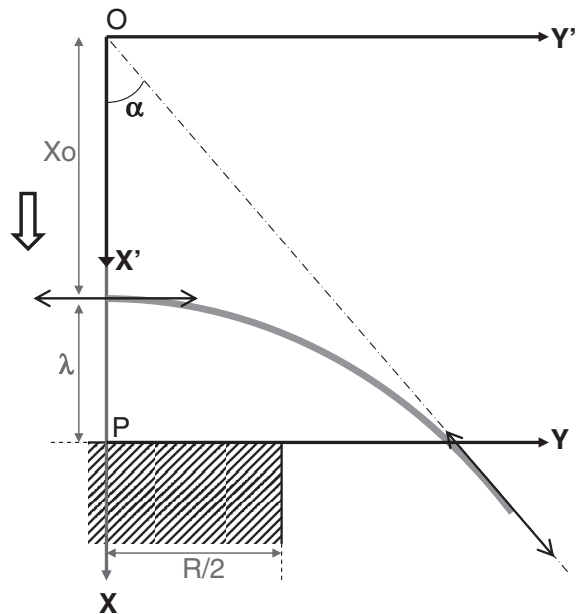

FIG. 6. Scheme of the hyperbolic fitting detachment curves with the obstacle (hatching), both asymptotes (double arrows), the equivalent Froude angle $\alpha$, and both axis systems $\left(O, X^{\prime}, Y^{\prime}\right)$ and $(P, X, Y)$.

The hyperbolic curve equation adapted from Ref. 12 is presented below. The shape of the fitting curve is based on two typical characteristics of the corresponding detached flow structures.

- They are normal to the free stream at their foremost point, that is, facing the center of the obstacle. The corresponding transverse asymptote is drawn on Fig. 6.

- They are asymptotic to the free stream Mach line at large distance from the obstacle. The corresponding asymptote is drawn on Fig. 6; its angle (named Froude angle) is analog to the Mach angle (equal to the semiangle of the Mach cone; see, for instance, Ref. 11) with $\tan \alpha=1 / \beta$ and $\beta=\sqrt{\mathrm{Fr}^{2}-1}$.

The most simple equation, which satisfies both asymptotes was proposed by Moeckel ${ }^{12}$ in the axis system $\left(O, X^{\prime}, Y^{\prime}\right)$ shown in Fig. 6, where $O$ is the intersection between the Froude line and the symmetry axis (see Fig. 6). This equation reads

$$
\beta Y^{\prime}=\sqrt{X^{\prime 2}-X_{o}^{2}},
$$

with $X_{o}$ as the distance between $O$ and the vertex of the flow structure (hydraulic jump or horseshoe vortex). When normalized by the obstacle width $R$, Eq. (8) yields

$$
\frac{\beta Y^{\prime}}{R}=\sqrt{\left(\frac{X^{\prime}}{R}\right)^{2}-\left(\frac{X_{o}}{R}\right)^{2}} .
$$

Attention should be made that the obstacle width defined here slightly differs from Moeckel ${ }^{12}$ consideration: $R=2 y_{S B}$.

Equation (9) contains only one unknown: $X_{o}$ as $\beta$ and $R$ are known for a given flow configuration. However, the prediction of location of both flow structures with regard to the obstacle requires to define the curve equation in a reference axis system linked to the obstacle. We then define a second axis system $(P, X, Y)$ in Fig. 6 with $P$ as the center of the upstream face of the obstacle. Equation (9) becomes

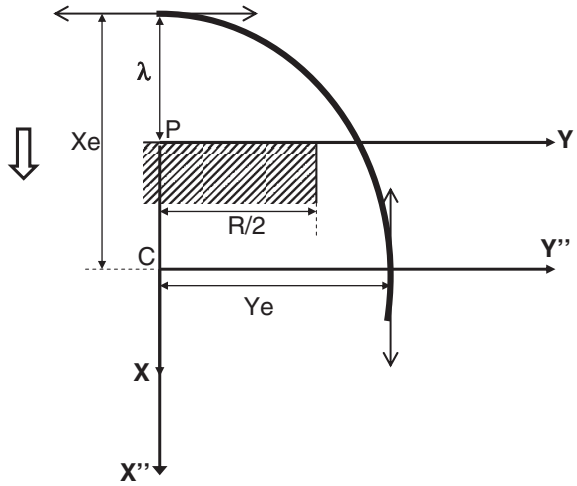

FIG. 7. Scheme of the elliptic fitting detachment curves with the obstacle (hatching), both asymptotes (double arrows), and both axis systems $\left(C, X^{\prime \prime}, Y^{\prime \prime}\right)$ and $(P, X, Y)$.

$$
\frac{\beta Y}{R}=\sqrt{\left(\frac{X+\lambda+X_{o}}{R}\right)^{2}-\left(\frac{X_{o}}{R}\right)^{2}} .
$$

In Eq. (10), $\beta$ and $X_{o} / R$ govern the shape (squeezing up or opening up) of the detachment curve, while $\lambda / R$ governs the detachment of this curve upstream from the obstacle: if $\lambda / R$ increases, the curve is moved away from the obstacle; if $\beta$ increases ( $\alpha$ decreases), the curve squeezes up; and if $X_{o} / R$ increases, the curve opens up. For this hyperbolic curve, the determination of each equation curve requires the determination of both the detachment length value $\lambda$ and the curve parameter $X_{o}$.

The elliptic curve equation is presented below. The ellipse is symmetric with regards to the flow axis facing the center of the obstacle. The center of the ellipse is noted as $C$, with $\left(C, X^{\prime \prime}, Y^{\prime \prime}\right)$ as the associated axis system, and its axes are noted as $X_{e}$ and $Y_{e}$ along $X^{\prime \prime}$ and $Y^{\prime \prime}$, respectively (see Fig. 7). Its equation simply reads

$$
\left(\frac{X^{\prime \prime}}{X_{e}}\right)^{2}+\left(\frac{Y^{\prime \prime}}{Y_{e}}\right)^{2}=1
$$

As for the previously described hyperbolic curve, the elliptic curve equation can be normalized by $R$ and written in the $(P, X, Y)$ axis system, and Eq. (11) becomes

$$
\left(\frac{Y_{e}}{X_{e}}\right)^{2}\left(\frac{X-X_{e}+\lambda}{R}\right)^{2}+\left(\frac{Y}{R}\right)^{2}=\left(\frac{Y_{e}}{R}\right)^{2} \text {. }
$$

For this elliptic curve, the determination of each equation curve requires the determination of three parameters: $\lambda, X_{e}$, and $Y_{e}$.

In the following, the subscript $H$ and $J$ will refer to the horseshoe vortex and hydraulic jump detachment curve, respectively. Consequently, for each separation flow configuration, four parameters are determined experimentally: $\left(\lambda_{H}, X_{o H}\right)$ and $\left(\lambda_{J}, X_{o J}\right)$. The very fair agreement between experimental and modeled detachment curves using Eq. (10) on Fig. 5(a) for a separation type flow confirms that the hyperbolic shape proposed by Moeckel ${ }^{12}$ holds not only for detached jumps but also for the horseshoe vortex in supercritical regime. Similarly, for each breaking flow configuration, five parameters are determined experimentally: $\left(\lambda_{H}, X_{e H}\right.$, and 

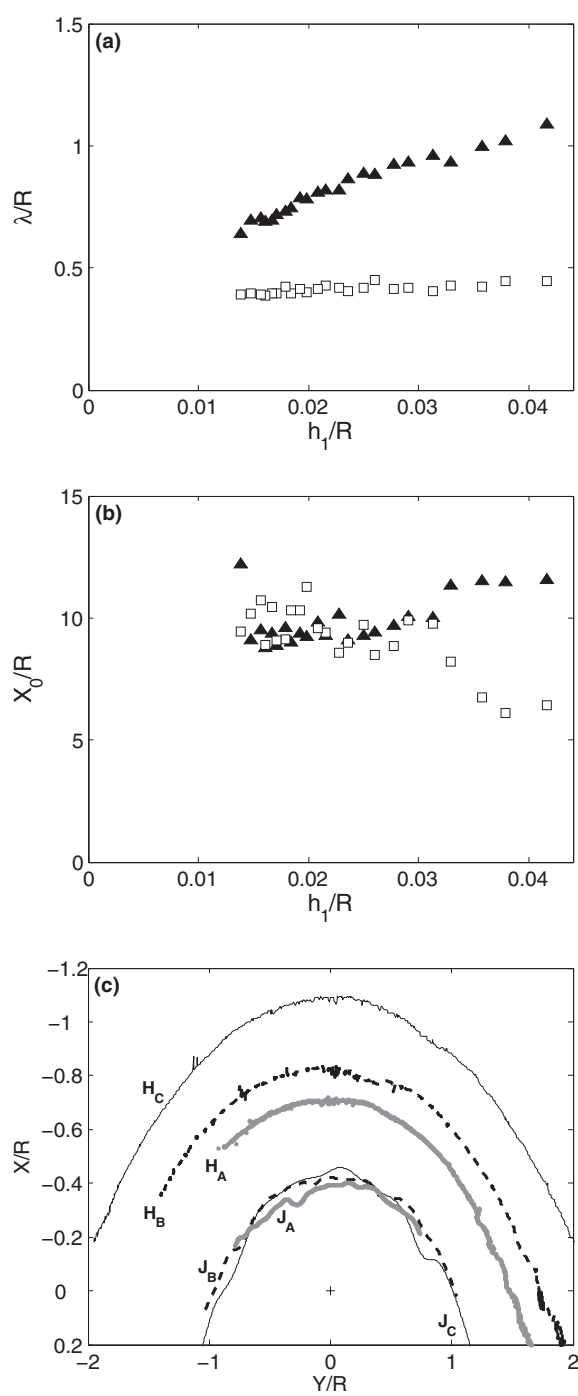

FIG. 8. (a) Normalized detachment lengths for the toe of the hydraulic jump ( $\square$ ) and the horseshoe vortex $(\boldsymbol{\Delta})$ as a function of the normalized upstream water depth for case $S 1$. (b) Best fitting parameter of the hydraulic jump ( $\square$ ) and horseshoe vortex $(\boldsymbol{\Delta})$ detachment curves for case $S 1$ using Eq. (10). (c) Toe of the jump $(J)$ and of the horseshoe vortex $(H)$ for $R=160 \mathrm{~mm}(\mathrm{~A})$, $R=110 \mathrm{~mm}(\mathrm{~B})$, and $R=60 \mathrm{~mm}(\mathrm{C})$ in case $S 1$.

$\left.Y_{e H}\right)$ for the elliptic horseshoe vortex curve and $\left(\lambda_{J}, X_{o J}\right)$ for the hyperbolic hydraulic jump curve. Figure 5(b) confirms the very fair agreement between experimental and modeled detachment curve of the hydraulic jump using Eq. (10) and of the horseshoe vortex using Eq. (12).

\section{A. Separation flow type}

In this section, the influence of the three normalized parameters of Eq. (7) on the horseshoe vortex and hydraulic jump detachment curves is investigated for the separation type configurations.

\section{Case S1: Influence of the normalized upstream water depth}

Keeping the same inflow conditions $\left(Q, h_{1}\right)$ and varying only the obstacle width $R$ permit to analyze the influence of the parameter $h_{1} / R$ on the detachment curves without alter-
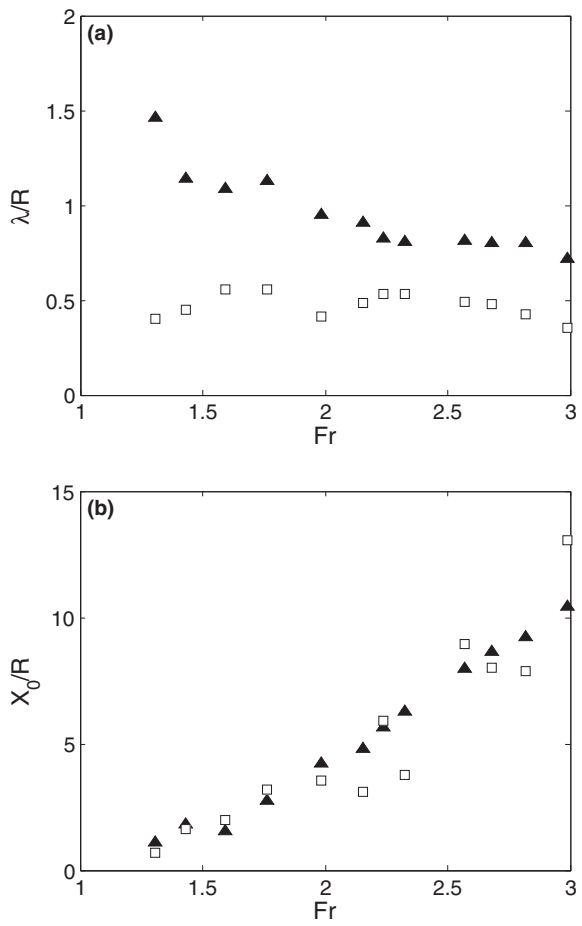

FIG. 9. [(a) and (b)] Same as Fig. 8 as a function of the upstream Froude number for case $S 2$ with $(\square)$ for the hydraulic jump and $(\boldsymbol{\Delta})$ for the horseshoe vortex.

ing the other parameters Fr and Re (see Table I). In such case, $\beta$ remains constant. Figure 8 (a) reveals that the normalized detachment length for the toe of the horseshoe vortex $\lambda_{H} / R$ increases quite linearly with $h_{1} / R$. Moreover, it appears that the normalized detachment length of the toe of the hydraulic jump $\lambda_{J} / R$ also increases but much more slightly. These results are confirmed in Fig. 8(c), where $\lambda_{H A} / R$ $<\lambda_{H B} / R<\lambda_{H C} / R$ and $\lambda_{J A} / R \lesssim \lambda_{J B} / R \lesssim \lambda_{J C} / R$. Moreover, Fig. 8(b) shows that $X_{O H} / R$ and $X_{O J} / R$ remain more or less constant at a value of about 10 for $h_{1} / R<0.03$. Oppositely, $X_{o H} / R$ increases and $X_{o J} / R$ decreases for higher $h_{1} / R$ values. $X_{o H} / R, X_{o J} / R$, and $\beta$ are quite similar for all configurations, which leads to very similar normalized detachment curves on Fig. 8(c), where only the normalized detachment lengths are altered. Finally, Fig. 8(c) confirms the hyperbolic shape of the toe of the hydraulic jump and of the horseshoe vortex in separation type

\section{Case S2: Influence of the Froude number}

Keeping $Q$ constant while changing $h_{1}$ and $R$ permits to vary $\operatorname{Fr}\left(\right.$ and $\beta$ ) without altering $\operatorname{Re}$ and $h_{1} / R$. Figure 9(a) shows that increasing the Froude number tends to decrease $\lambda_{H} / R$ but does not change much $\lambda_{J} / R$ (which remains quite constant at an average value of 0.5$)$.

Moreover, Fig. 9(b) shows that $X_{o H} / R$ and $X_{o J} / R$ remain more or less equal to each other and increase as Fr increases.

\section{Case S3: Influence of the Reynolds number}

Figures 10(a) and 10(b) show that the value of Re has very little influence on the normalized detachment lengths $\lambda_{H} / R$ and $\lambda_{J} / R$ and the curve parameters $X_{o H} / R$ and $X_{o J} / R$. 

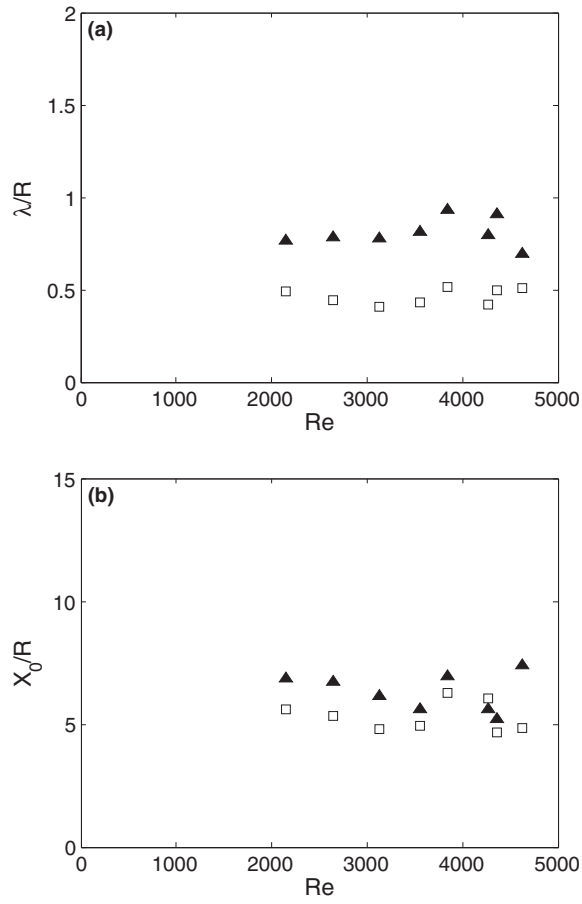

FIG. 10. [(a) and (b)] Same as Fig. 8 as a function of the Reynolds number for case $S 3$ with $(\square)$ for the hydraulic jump and $(\boldsymbol{\Lambda})$ for the horseshoe vortex.

Consequently, as $\beta$ is kept constant ( $\mathrm{Fr}$ is constant) for all flow configurations, all curves are similar. Let us note, however, that the absolute values of $\lambda_{H}, \lambda_{J}, X_{o H}$, and $X_{o J}$ are strongly affected by the Reynolds number value; only their normalized values remain constant.

\section{B. Breaking flow type}

In this section, the influence of the three normalized parameters of Eq. (7) on the horseshoe vortex and hydraulic jump detachment curves is investigated for the breaking type configurations where the hydraulic jump is fitted by Eq. (10) and the horseshoe vortex by Eq. (12).

\section{Case B1: Influence of the normalized upstream water depth}

Figure 11(a) reveals that $\lambda_{J} / R$ increases rapidly with increasing $h_{1} / R$ value while $\lambda_{H} / R$ increases more slightly. For the hydraulic jump, Fig. 11(b) shows that the curve parameter $X_{o J} / R$ remains more or less constant. As $\beta$ also remains constant for all flows, the shapes of the hydraulic jump detachment curves are kept constant, and only their detachment lengths $\lambda_{J} / R$ vary. For the horseshoe vortex detachment curves, the parameters $X_{e H} / R$ and $Y_{e H} / R$ also remain quite constant for all $h_{1} / R$ values on Fig. 11(c), and Fig. 11(d) confirms that only their detachment lengths $\lambda_{H} / R$ vary. Finally, Fig. 11(d) confirms the hyperbolic shape of the toe of the hydraulic jump and the elliptic shape of the toe of the horseshoe vortex in breaking type.
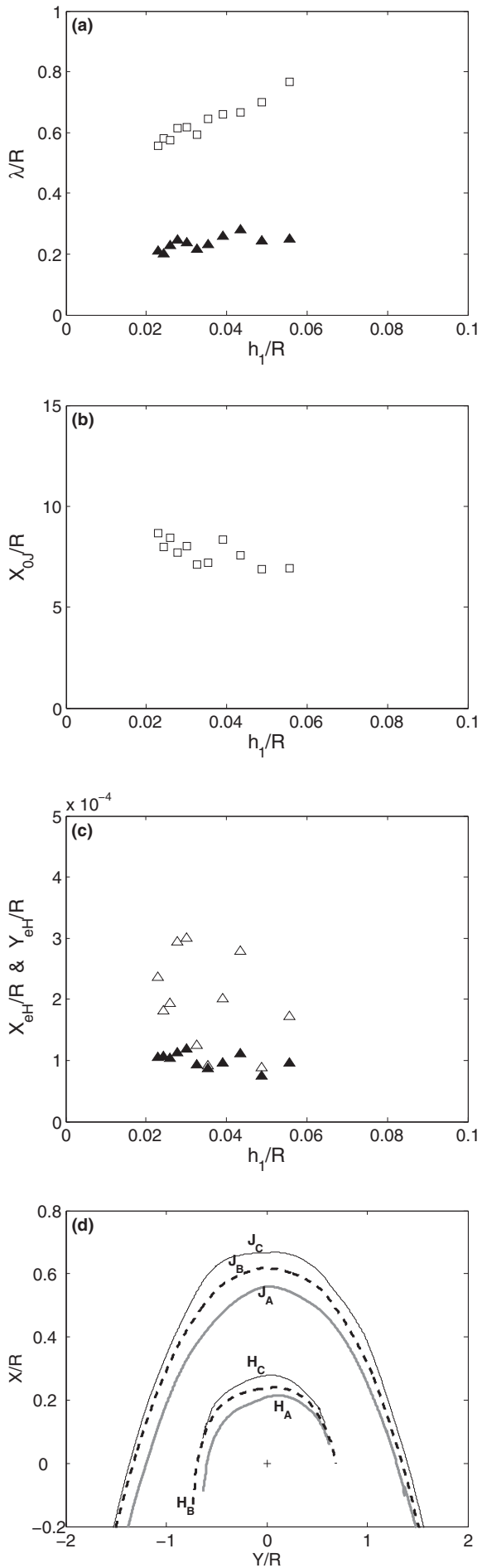

FIG. 11. (a) Normalized detachment lengths for the toe of the hydraulic jump ( $\square$ ) and the horseshoe vortex $(\boldsymbol{\Delta})$ as a function of the normalized upstream water depth for case $B 1$. (b) Best fitting $X_{o J} / R$ parameter of the hydraulic jump detachment curve for case $B 1$ using Eq. (10). (c) Best fitting $X_{e H} / R(\triangle)$ and $Y_{e H} / R(\Delta)$ parameters of the horseshoe vortex detachment curve for case $B 1$ using Eq. (12). (d) Toe of the jump $(J)$ and of the horseshoe vortex $(H)$ for $R=170 \mathrm{~mm}(\mathrm{~A}), R=130 \mathrm{~mm}(\mathrm{~B})$, and $R=90 \mathrm{~mm}(\mathrm{C})$ in case $B 1$.

\section{Case B2: Influence of the Froude number}

Figure 12(a) shows that $\lambda_{H} / R$ and $\lambda_{J} / R$ decrease with increasing Froude number. For the hydraulic jump detach- 

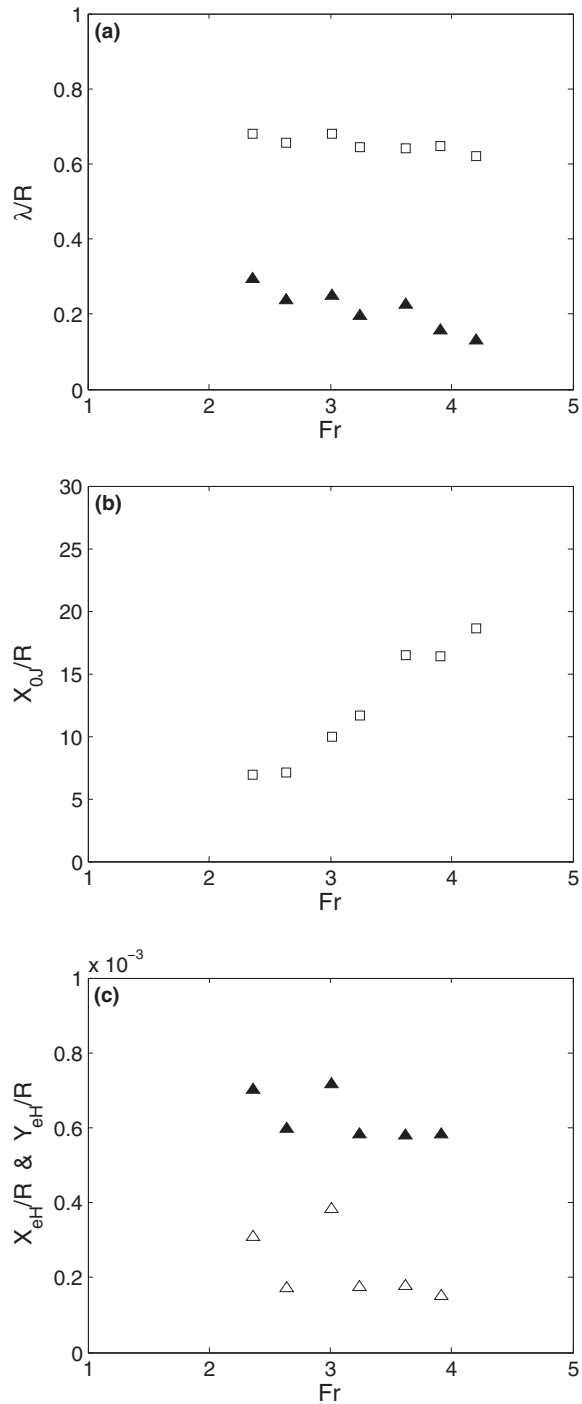

FIG. 12. [(a)-(c)] Same as Fig. 11 as a function of the upstream Froude number for case $B 2$ with $(\square)$ for the hydraulic jump and $(\mathbf{\Lambda})$ for the horseshoe vortex.

ment curves, $X_{o J} / R$ increases with increasing $\operatorname{Fr}$ [see Fig. 12(b)].For the horseshoe vortex detachment curves, the parameters $X_{e H} / R$ and $Y_{e H} / R$ slightly decrease with increasing Fr [see Fig. 12(c)].

\section{Case B3: Influence of the Reynolds number}

As for the separation type, Figs. 13(a) and 13(b) show that Re has very little influence on the hydraulic jump characteristics $\lambda_{J} / R$ and $X_{o J} / R$. For the horseshoe vortex curves, Figs. 13(a) and 13(c) show that $\lambda_{H} / R, X_{e H} / R$, and $Y_{e H} / R$ also remain quite constant for all tested Re values.

\section{Discussions}

The influence of the three parameters from Eq. (7) on the detachment curves of the horseshoe vortex and the hydraulic jump is summarized in Table II. Regarding the detachment lengths, quite similar tendencies are encountered for separation and breaking flow types.
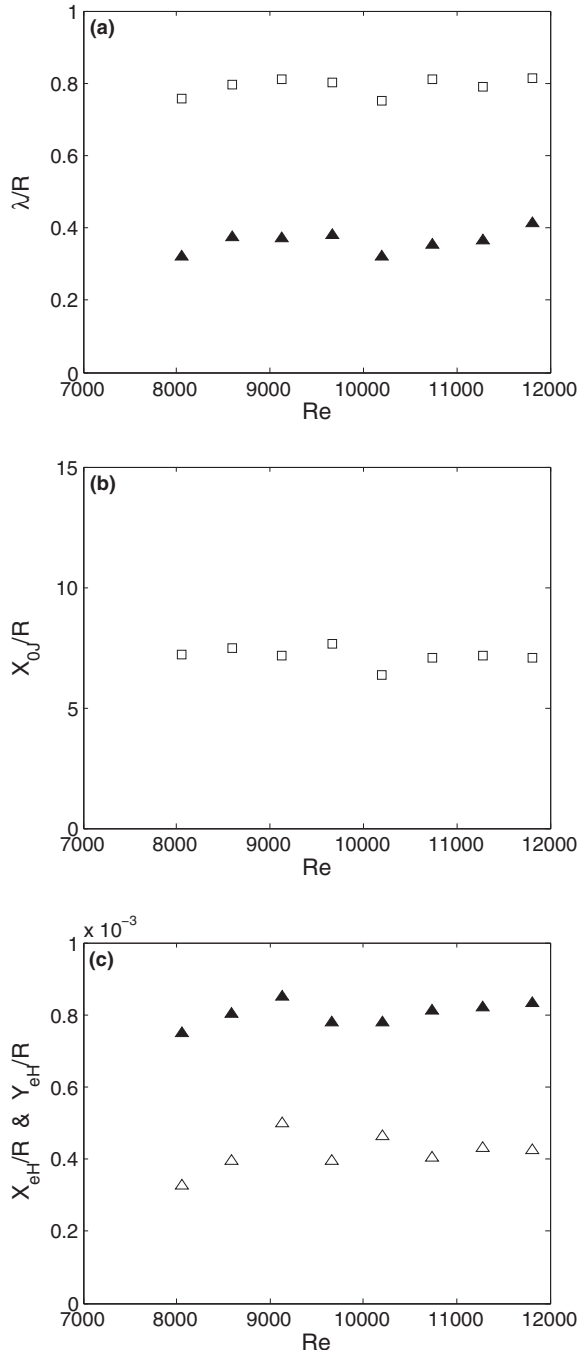

FIG. 13. [(a)-(c)] Same as Fig. 11 as a function of the Reynolds number for case $B 3$ with $(\square)$ for the hydraulic jump and $(\boldsymbol{\Delta})$ for the horseshoe vortex.

- Increasing the Froude number of the inflow (Fr) tends to decrease the normalized detachment length of both the hydraulic jump $\left(\lambda_{J} / R\right)$ and the horseshoe vortex $\left(\lambda_{H} / R\right)$.

- Increasing the normalized water depth of the inflow $\left(h_{1} / R\right)$ tends to increase $\lambda_{J} / R$ and $\lambda_{H} / R$.

- Altering the Reynolds number $(\mathrm{Re})$ does not affect the detachment lengths.

Regarding the shape of the detachment curves, the influence of the three parameters on the hydraulic jump shape parameter $X_{o J} / R$ is similar for both flow types (only Fr has an impact on $X_{o J} / R$ ). Similarly, for the horseshoe vortex detachment curve, only Fr impacts the shape parameters $X_{o} / R$, $X_{e} / R$, and $Y_{e} / R$.

The influence of the three parameters from Eq. (7) on the normalized detachment length of the hydraulic jump $\left(\lambda_{J} / R\right)$ can be explained using the same approach as proposed by Moeckel $^{12}$ for a supersonic air flow interacting with a squared-shape obstacle. As shown in Fig. 14, Moeckel ${ }^{12}$ applied the law of mass conservation between the supersonic 
TABLE II. Summary of the tendencies observed in the experimental analysis.

\begin{tabular}{|c|c|c|c|c|c|c|}
\hline & \multicolumn{3}{|c|}{ Separation flow type } & \multicolumn{3}{|c|}{ Breaking flow type } \\
\hline & $\lambda / R$ & $X_{o} / R$ & $\begin{array}{l}R^{2} \\
(\%)\end{array}$ & $\lambda / R$ & $X_{o} / R$ or $X_{e} / R, \quad Y_{e} / R$ & $\begin{array}{c}R^{2} \\
(\%)\end{array}$ \\
\hline \multirow[t]{2}{*}{ Increasing $h_{1} / R$} & $\lambda_{H} / R \uparrow$ & $X_{o H} / R \approx$ & 99.4 & $\lambda_{H} / R \uparrow$ & $X_{e H} / R \approx, \quad Y_{e H} / R \approx$ & 98.8 \\
\hline & $\lambda_{J} / R \uparrow$ & $X_{o J} / R \approx$ & 98.3 & $\lambda_{J} / R \uparrow$ & $X_{o J} / R \approx$ & 99.7 \\
\hline \multirow[t]{2}{*}{ Increasing Fr } & $\lambda_{H} / R \downarrow$ & $X_{o H} / R \uparrow$ & 98.9 & $\lambda_{H} / R \downarrow$ & $X_{e H} / R \downarrow, \quad Y_{e H} / R \downarrow$ & 98.3 \\
\hline & $\lambda_{J} / R \approx$ & $X_{o J} / R \uparrow$ & 97.0 & $\lambda_{J} / R \downarrow$ & $X_{o J} / R \uparrow$ & 99.6 \\
\hline \multirow[t]{2}{*}{ Increasing $\mathrm{Re}$} & $\lambda_{H} / R \approx$ & $X_{o H} / R \approx$ & 99.4 & $\lambda_{H} / R \approx$ & $X_{e H} / R \approx, \quad Y_{e H} / R \approx$ & 98.7 \\
\hline & $\lambda_{J} / R \approx$ & $X_{o J} / R \approx$ & 96.4 & $\lambda_{J} / R \approx$ & $X_{o J} / R \approx$ & 99.9 \\
\hline
\end{tabular}

flow entering the control volume with velocity $U_{1}$ and density $\rho_{1}$, reaching the subsonic conditions $\left(\rho_{2}\right)$ and leaving it with sonic velocity $U_{2}^{*}$ and density $\rho_{2}^{*}$. The angle $\eta$ is defined as the angle between the sonic line (straight line of length $L$ on Fig. 14) and the direction of the obstacle face. The mass conservation law reads

$$
\rho_{1} U_{1}\left(\frac{R}{2}+L \cos \eta\right)=\rho_{2}^{*} U_{2}^{*} L
$$

where $L=L(\lambda, \eta)$. Following the perfect gas assumption with $M$ as the incoming Mach number and $T$ as the absolute temperature, Eq. (13) reads

$$
\rho_{1} M \sqrt{T_{1}}\left(\frac{R}{2}+L \cos \eta\right)=\rho_{2}^{*} \sqrt{T_{2}^{*}} L .
$$

Finally, we obtain

$$
\frac{L / R}{1 / 2+L \cos \eta / R}=\frac{\rho_{1}}{\rho_{2}^{*}} M \sqrt{\frac{T_{1}}{T_{2}^{*}}} .
$$

Moreover, in Eq. (15), $\rho_{1} / \rho_{2}^{*}$ and $T_{1} / T_{2}^{*}$ depend only on $M$ according to the shock relation (from 1 to 2 ) and the isentropic flow relation (from 2 to $2^{*}$ ), while $\lambda, \eta$, and $L$ are related through the hyperbolic curve equation, which depends on $M$ following the Mach angle expression. As a consequence, Eq. (15) reveals that $\lambda_{J} / R$ depends only on $M$, and Moeckel $^{12}$ analytically showed that $\lambda_{J} / R$ decreases when $M$ increases.

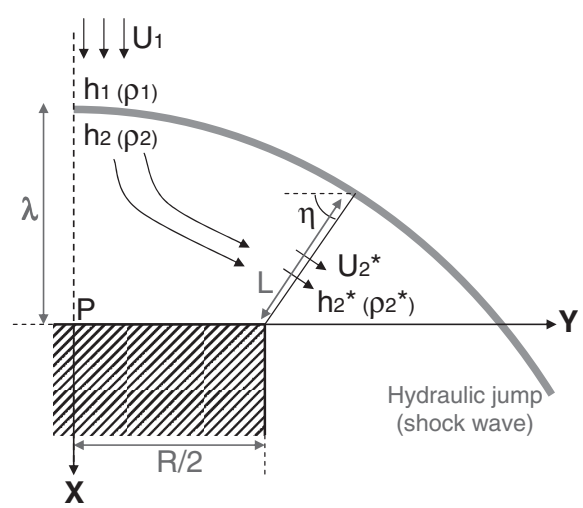

FIG. 14. Simplified scheme of the mass conservation analysis following Moeckel (Ref. 12) works where $h$ refers to water depths and $\rho$ refers to air density in wind tunnel configurations.
Applying this analysis to our hydraulic configuration where (i) the density of the air flow $(\rho)$ is replaced by the water depth $(h)$, (ii) the incoming Mach number $M$ is replaced by the incoming Froude number Fr, (iii) the shock wave becomes an hydraulic jump, and (iv) the width of the hydraulic jump remains infinitely thin as for the shock wave, it reads

$$
h_{1} U_{1}\left(\frac{R}{2}+L \cos \eta\right)=h_{2}^{*} U_{2}^{*} L
$$

and thus

$$
h_{1}^{3 / 2} \operatorname{Fr}\left(\frac{R}{2}+L \cos \eta\right)=h_{2}^{* 3 / 2} L
$$

and

$$
\frac{L / R}{1 / 2+L \cos \eta / R}=\operatorname{Fr}\left(\frac{h_{1}}{h_{2}^{*}}\right)^{3 / 2} .
$$

In Eq. (18), $h_{1} / h_{2}^{*}$ depends only on Fr through the oblique jump water depth equation (from 1 to 2 ) and the head conservation (between 2 and $2^{*}$ ). As in the previous case, $\lambda$, $\eta$, and $L$ are related through the hyperbolic curve equation, which depends on Fr following the Froude angle expression. As a consequence, it appears that $\lambda_{J} / R$ depends only on $\mathrm{Fr}$, and we experimentally showed that $\lambda_{J} / R$ decreases when $\mathrm{Fr}$ increases (see Table II).

Up to now, the hydraulic jump was considered as an infinitely thin flow structure (as it is the case for the shock wave in Moeckel ${ }^{12}$ analysis). However, it is well known that the hydraulic jump has a non-negligible width, and that for a fixed upstream Froude number Fr, this width is strongly related to the upstream water depth $h_{1}$. The change of hydraulic jump width modifies the limits of the control volume in Fig. 14, which becomes unknown and thus affects the relation between $\eta, L$, and $\lambda_{J}$. Consequently, altering $h_{1} / R$ modifies $\lambda_{J} / R$ as observed on our experimental results (see Table II).

As in Moeckel ${ }^{12}$ work, the Reynolds number of the inflow is not involved in the mass conservation analysis as it does not alter neither the angle $\eta$ nor the shock relation and the head conservation law. Consequently, the Reynolds number does not affect the detachment length of the hydraulic jump $\lambda_{J} / R$ as observed in Table II. 


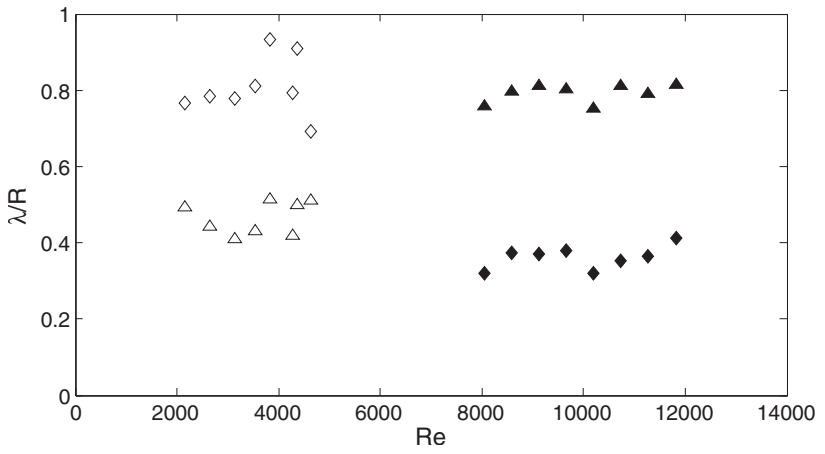

FIG. 15. $\lambda_{J} / R$ (triangles) and $\lambda_{H} / R$ (lozenges) for separation type (in white) and breaking type (in black) flows as a function of the Reynolds number (from Figs. 10 and 13).

In separation type flow configurations, the impact of the three parameters of Eq. (7) on the detachment length of the horseshoe vortex can be easily derived from their impact on the hydraulic jump detachment curve discussed above. Indeed, as explained in the text, the behavior of the horseshoe vortex follows the hydraulic jump behavior. Nevertheless, in breaking type flow configuration, the location of the horseshoe vortex depends on both the hydraulic jump location and the backwater curve of the free surface facing the obstacle. We showed in the text that in such breaking type, the horseshoe vortex curve becomes elliptic, and the previous equations based on Moeckel $^{12}$ mass conservation analysis cannot be applied. Finally, the influence of the Morton number Mo on both the hydraulic jump and horseshoe vortex curves was investigated by adding soap to the water in order to decrease the surface tension and thus increase the Morton number. We observed that such modification of the fluid had no influence on the location of the detached curves and thus that Mo had no impact in Eq. (5).

Another interesting aspect that should be discussed is the following. With turbulent inflow conditions (breaking type), the boundary layer separation occurs far downstream, in the near obstacle region and the hydraulic jump location is governed by backwater considerations. Oppositely with laminar inflow condition (separation type), the boundary layer separation occurs much further upstream and tends to increase the inflow water depth, which causes the hydraulic jump to be "pushed" further downstream in the near obstacle region. When comparing cases $S 3$ and $B 3$ with similar Fr and $h_{1} / R$ values on Fig. 15, it appears that the normalized detachment lengths are opposed one to the other: $\lambda_{H} / R$ for separation type flows is of the same order of magnitude as $\lambda_{J} / R$ for breaking type flows, and similarly $\lambda_{H} / R$ for breaking type flows is of the same order of magnitude as $\lambda_{J} / R$ for separation type flows.

\section{CONCLUSION}

This paper details the flow pattern that occurs upstream from an obstacle as a supercritical open channel flow interacts with an emerging rectangular shape obstacle. An experimental setup using a transparent smooth and wide water table was deployed using rectangular shape obstacles permit- ting a wide range of width variations. As the inflow approaches the obstacle, a transition from supercritical to subcritical regime occurs and the flow eventually stops at the obstacle face. This transition is performed through a hydraulic jump, which is normal to the flow axis facing the center of the obstacle and is oblique on the sides of the obstacle. Moreover, as the flow depth increases from uniform depth upstream to the stagnation point on the obstacle facade, the reverse pressure gradient leads to a boundary layer separation and a horseshoe vortex is observed. Both flow structures (the hydraulic jump in the near-surface region and the horseshoe vortex in the near-bed region) are detached upstream from the obstacle and their detachment length varies depending on the flow and obstacle characteristics. Two main flow types can then be observed. First, for a fully turbulent inflow, the detachment length of the hydraulic jump exceeds the one of the horseshoe vortex; this flow type is named "breaking type." Oppositely, for a laminar inflow, the detachment length of the horseshoe vortex exceeds the one of the hydraulic jump and the flow type is named "separation type." Experimental measurements confirmed that the Reynolds number is the main parameter governing the occurrence of the flow types.

Experimental methodologies were developed in order to detect the shape and location of the curve corresponding to the foot of both flow structures for each flow type. The measurements revealed that for both types, the hydraulic jump detachment curve is constrained by two asymptotes: one normal to the flow axis in front of the obstacle and a second on its sides, making a specific angle (Froude angle) with the flow axis. In separation type, the horseshoe vortex takes place in a region of supercritical flow regime and follows the same hyperbolic shape as the hydraulic jump, while in breaking type, the horseshoe vortex that takes place in a region of subcritical flow regime follows an elliptic shape.

The dimensional analysis revealed that three parameters can influence the detachment curves: the upstream Froude and Reynolds numbers and the upstream water depth normalized by the obstacle thickness. The influence of these parameters was investigated experimentally by keeping two parameters constant and varying the third one. We showed that the influence of these parameters is quite similar for both flow structures and for both flow types: increasing the Froude number of the inflow tends to decrease the normalized detachment lengths and increasing the normalized upstream water depth tends to increase these lengths, while the impact of the Reynolds number on the detachment lengths remains very limited.

Finally, we identified two different processes responsible for the boundary layer detachment: in breaking type, the detachment is caused by the backwater effect facing the obstacle with a water depth increase in subcritical flow conditions, and in separation type, the detachment is caused by the sudden water depth increase in the hydraulic jump as mentioned by Lennon and Hill ${ }^{26}$ and Mignot and Cienfuegos. ${ }^{27}$ 


\section{ACKNOWLEDGMENTS}

The authors would like to thank F. Chabardes, N. Gonima, and I. Renaud for participating to preliminary experiments. The authors are also grateful to L. Kaci, R. Sadani, and A. Zelez for their technical support. Moreover, the authors are grateful to an anonymous referee who suggested the ellipse fitting in Sec. VII, "Measurement of Detachment Curves."

${ }^{1}$ W. A. Eckerle and J. K. Awad, "Effect of freestream velocity on the three-dimensional separated flow region in front of a cylinder," ASME Trans. J. Fluids Eng. 113, 37 (1991).

${ }^{2}$ R. J. Pattenden, S. R. Turnock, and X. Zhang, "Measurement of the flow over a low-aspect-ratio cylinder mounted on a ground plane," J. Eng. Mech. 39, 10 (2005).

${ }^{3}$ C. J. Baker, "The turbulent horseshoe vortex," J. Wind. Eng. Ind. Aerodyn. 6, 9 (1980).

${ }^{4}$ F. Ballio, C. Bettoni, and S. Franzetti, "A survey of time-averaged characteristics of laminar and turbulent horseshoe vortices," ASME Trans. J. Fluids Eng. 120, 233 (1998).

${ }^{5}$ B. Dargahi, "The turbulent flow field around a circular cylinder," Exp. Fluids 8, 1 (1989).

${ }^{6}$ B. Sahin, N. A. Ozturk, and H. Akilli, "Horseshoe vortex system in the vicinity of the vertical cylinder mounted on a flat plate," J. Fluid Mech. 18, 57 (2007).

${ }^{7}$ W. H. Graf and B. Yulistiyanto, "Experiments of flow around a cylinder; the velocity and vorticity fields," J. Eng. Mech. 36, 637 (1998).

${ }^{8}$ F. Ahmed and N. Rajaratnam, "Flow around bridge piers," J. Hydraul. Eng. 124, 288 (1998)

${ }^{9}$ A. Roulund, M. Sumer, J. Fredsoe, and J. Michelsen, "Numerical and experimental investigation of flow and scour around a circular pile," J. Fluid Mech. 534, 351 (2005).

${ }^{10}$ M. A. Sadeque, N. Rajaratnam, and M. R. Loewen, "Flow around cylinders in open channels," J. Eng. Mech. 134, 60 (2008).

${ }^{11}$ A. H. Shapiro, The Dynamics and Thermodynamics of Compressible Fluid Flow (Ronald, New York, 1953), pp. 881-888.
${ }^{12}$ W. E. Moeckel, "Approximate method for predicting form and location of detached shock waves ahead of planar or axially symmetric bodies," Technical Note 1921, National Advisory Committee for Aeronautics, 1949, p. 32 .

${ }^{13}$ T. Mizukaki, "Detached shock waves around cylinders flying at Mach number ranging from 1 to 2," J. Visualization 11, 133 (2008).

${ }^{14} \mathrm{P}$. Gerlinger and M. Aigner, "Multigrid simulations of detached shock waves," Int. J. Numer. Methods Fluids 44, 1045 (2004).

${ }^{15}$ L. K. Forbes and L. W. Schwartz, "Supercritical flow past blunt bodies in shallow water," Z. Angew. Math. Phys. 32, 314 (1981).

${ }^{16}$ Q. Jiang and R. B. Smith, "V-waves, bow shocks, and wakes in supercritical hydrostatic flows,” J. Fluid Mech. 406, 27 (2000).

${ }^{17}$ A. T. Ippen, "Mechanics of supercritical flow: 1st paper of high velocity flow in open channels: A symposium," Transactions ASCE 116, 268 (1951).

${ }^{18}$ A. Defina and F. M. Susin, "Multiple states in open channel flow, in vorticity and turbulence effects in fluid structures interactions," Advances in Fluid Mechanics, edited by M. Brocchini and F. Trivellato (WIT Press, Southampton, UK, 2006), pp. 105-130.

${ }^{19}$ H. Tennekes and J. L. Lumley, A First Course in Turbulence (MIT Press, Cambridge, 1972).

${ }^{20}$ J. E. Richardson and V. G. Panchang, "Three-dimensional simulation of scour-inducing flow at bridge piers," J. Hydrol. Eng. 124, 530 (1998).

${ }^{21}$ W. H. Hager, "Impact hydraulic jump," J. Hydrol. Eng. 102, 633 (1994).

${ }^{22}$ C. J. Wood, IAHR Hydraulic Structure Design Manual No. 4, Hydraulic Design Considerations (Balkema, Rotterdam, The Netherlands, 1991).

${ }^{23}$ H. Chanson, Air Bubble Entrainment in Free Surface Turbulent Shear Flows (Academic, London, 1997).

${ }^{24}$ K. G. Ranga Raju, G. L. Asawa, and H. K. Misra, "Flow establishment length in rectangular channels and ducts," J. Hydrol. Eng. 126, 533 (2000).

${ }^{25}$ J. H. Agui and J. Andreopoulos, "Experimental investigation of a threedimensional boundary layer flow in the vicinity of an upright wall mounted cylinder," ASME Trans. J. Fluids Eng. 114, 566 (1992).

${ }^{26}$ J. Lennon and D. Hill, "Particle image velocity measurements of undular and hydraulic jumps,” J. Hydrol. Eng. 132, 1283 (2006).

${ }^{27}$ E. Mignot and R. Cienfuegos, "Energy dissipation and turbulent production in weak hydraulic jumps," J. Hydrol. Eng. 136, 116 (2010). 